\title{
Resultados alejados de la cirugía reparadora de la insuficiencia mitral degenerativa
}

\author{
Ricardo Zalaquett S, Cristóbal Camplá Ca, Samuel Córdova A, \\ Sandra Braun J, G astón Chamorro S, Manuel Irarrázaval LI, \\ Sergio Morán V, Pedro Becker R, Iván G odoy J, \\ Fernando Yáñez D.
}

\section{Long-term results of mitral valve repair for degenerative mitral insufficiency}

Background: Mitral valve repair is probably the procedure of choice for the surgical treatment of degenerative mitral insufficiency. Aim: To evaluate the late results of mitral valve repair in degenerative mitral insufficiency. Patients and method: The records of 88 patients who underwent mitral valve repair for degenerative mitral insufficiency from December 1991 through June 2002 were reviewed. Mean age was 59.9 years (range 22 to 82). At least moderate mitral insufficiency was present in every patient. Mean left atrial diameter was $55 \mathrm{~mm}$ and mean end diastolic and end systolic left ventricular diameters were 61 and $37 \mathrm{~mm}$ respectively. Results: The most common underlying lesion was ruptured chordae tendineae (66\%) and posterior leaflet prolapse (68\%). The surgical procedure most frecuently performed was quadrilateral resection of the posterior leaflet (68\%). A Carpentier-Edwards ring was placed in $97 \%$ of patients. An associated procedure was performed in $34 \%$. Operative mortality was $2.3 \%$. A complete follow up was obtained in $93 \%$ of cases with a mean of $54 \pm 36$ months. Overall survival rate was $98 \%$ at one year, $88 \%$ at 5 and $82 \%$ at 10 years. Free of cardiac death rates were $94 \%$ at 5 and $89 \%$ at 10 years. Only 2 patients were reoperated during follow up, resulting in a 98\% reoperation free rate follow up. Functional class improved in all patients at the end of follow up. Late echocardiographic evaluation showed absent or minimal mitral regurgitation in $83 \%$ and mild mitral regurgitation in $17 \%$. Conclusion: Good late results have been obtained with mitral valve repair, avoiding the inconveniencies of prosthetic replacement. Therefore, mitral valve repair should be the procedure of choice to treat degenerative mitral insufficiency (Rev Méd Chile 2003; 131: 1355-64).

(Key Words: Mitral valve insufficiency)

Recibido el 11 de junio, 2003. Aceptado en versión corregida el 13 de agosto, 2003.

Departamento de Enfermedades Cardiovasculares, Facultad de Medicina, Pontificia Universidad Católica de Chile.

aAyudante alumno.

Correspondencia a: Dr. Ricardo Zalaquett S. Marcoleta 367, 6o piso. Teléfonos: 6333030-6863231. Fax: 6390108. E mail: rzalaque@med.puc.cl 
$\mathrm{E}^{\mathrm{n}}$ la actualidad la cirugía reconstructora es el procedimiento de elección para la insuficiencia de la válvula mitral, puesto que permite la conservación de la integridad del aparato valvular mitral y con esto preserva la geometría de la cavidad ventricular izquierda, lo que se traduce en una mantención y mejoría de la función del ventrículo izquierdo ${ }^{1}$. Sin embargo, fue necesario que pasara el tiempo para que la cirugía reparadora de la insuficiencia mitral fuera universalmente aceptada como el procedimiento de elección para esta enfermedad valvular.

Por nuestra parte, en 1991 iniciamos nuestra experiencia en cirugía reparadora de la insuficiencia mitral de acuerdo a los principios y técnicas de Carpentier, con control ecocardiográfico transesofágico intraoperatorio. En 1994 presentamos nuestros resultados inmediatos, con énfasis en los aspectos quirúrgicos ${ }^{2}$, y, en 1996 nuestros resultados precoces, dando particular importancia a los aspectos ecocardiográficos perioperatorios ${ }^{3}$. En 1999 comunicamos nuestros resultados tardíos, incluyendo todos los pacientes en los que se efectuó una reparación de la válvula mitral, dando especial atención a los aspectos de sobrevida y resultados ecocardiográficos ${ }^{4}$.

En esta oportunidad presentamos los resultados alejados, clínicos y ecocardiográficos, de la cirugía reparadora de la insuficiencia de la válvula mitral secundaria exclusivamente a una degeneración mixomatosa de ésta.

\section{PACIENTES Y MÉTODO}

Pacientes. Entre diciembre de 1991 y junio de 2002, 166 pacientes con insuficiencia mitral de diferentes etiologías tuvieron un procedimiento reparador de su válvula mitral, como se detalla en la Tabla 1. De éstos, 88 (53\%) tenían una insuficiencia mitral degenerativa y constituyen la población en estudio. La edad promedio fue 59,9 $\pm 13,2$ años (22 a 82 años) y 48 (55\%) eran hombres. Por otra parte, estos 88 pacientes representaron el $71 \%$ del total de 124 pacientes con insuficiencia mitral degenerativa operados durante este período. Los otros 36 tuvieron un reemplazo valvular. La Figura 1 representa cómo durante este período la reparación fue progresivamente superando al reemplazo protésico como tratamiento quirúrgico de la insuficiencia mitral degenerativa.

Tabla 1. Etiología de insuficiencia mitral en $\mathbf{1 6 6}$ pacientes con reparación valvular mitral operados entre diciembre de 1991 y junio 2002

\begin{tabular}{|lcr|}
\hline Etiología & $\mathrm{N}^{0}$ pacientes & $\%$ \\
\hline Degenerativa & 88 & 53 \\
Isquémica & 40 & 24 \\
Reumática & 17 & 10 \\
Funcional & 12 & 7 \\
Infecciosa & 6 & 4 \\
Otra & 3 & 2 \\
\hline
\end{tabular}

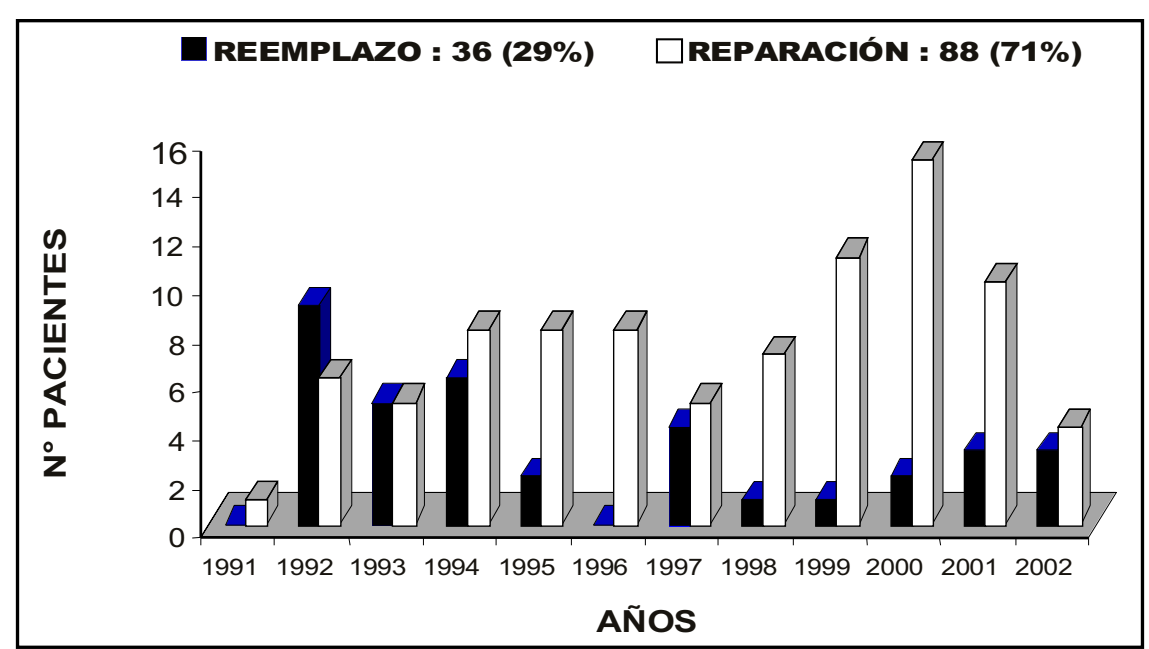

Figura 1. Comparación entre reemplazo y reparación valvular mitral por insuficiencia mitral degenerativa efectuados entre diciembre 1991 y junio 2002 (total de pacientes: 124). En 30 pacientes con recambio mitral se usó una prótesis mecánica y en 6 biológica. 
Características clínicas y ecocardiográficas. El $12 \%$ de los pacientes se encontraban en capacidad funcional (CF) I, $25 \%$ en II, $51 \%$ en III y $12 \%$ en IV, al momento de la cirugía. Así, 37\% de los pacientes estaban asintomáticos o poco sintomáticos cuando se efectuó la indicación quirúrgica.

Ecocardiográficamente todos los pacientes tenían insuficiencia mitral moderada (3+) o severa (4+). El diámetro auricular izquierdo promedio fue $55 \mathrm{~mm} \pm$ $11 \mathrm{~mm}$. El diámetro diastólico promedio del ventrículo izquierdo fue $61 \mathrm{~mm} \pm 7,6 \mathrm{~mm}$ y el sistólico 37 $\mathrm{mm} \pm 9,5 \mathrm{~mm}$. La presión sistólica de arteria pulmonar fue en promedio $47 \mathrm{~mm} \mathrm{Hg} \pm 10 \mathrm{~mm} \mathrm{Hg}$.

Técnica quirúrgica. Todos los pacientes fueron operados con circulación extracorpórea con hipotermia sistémica entre $28^{\circ}$ y $30^{\circ} \mathrm{C}$. La protección miocárdica se efectuó con solución cardioplégica cristaloide, con una dosis inicial por vía anterógrada y continuada luego por vía retrógrada cada 20 $\min ^{5}$. El abordaje a la válvula mitral fue a través de una auriculotomía izquierda transeptal y superior $^{6}$. La reparación de la válvula mitral se efectuó de acuerdo a los principios y técnicas establecidas por Carpentier 7 , con control ecocardiográfico transesofágico intraoperatorio.

Seguimiento. Los pacientes fueron contactados personal o telefónicamente entre agosto y septiembre de 2002. Asimismo, se revisó el último ecocardiograma disponible luego del alta hospitalaria. Finalmente, se solicitó información al Registro Civil e Identificación de Chile respecto a mortalidad.

Análisis estadístico. Los datos se expresan como promedio \pm desviación estándar y la sobrevida se analizó mediante el método de Kaplan-Meier. Para las comparaciones de CF y parámetros ecocardiográficos pre y post reparación mitral alejados se utilizó el test no paramétrico de sumas de rangos de Wilcoxon, considerándose significativo un valor $\mathrm{p}<0,05$. Para la presentación de los resultados se siguieron las recomendaciones del comité conjunto de la American Association for Thoracic Surgery y la Society of Thoracic Surgeons $s^{8}$.

\section{RESULTADOS}

Hallazgos intraoperatorios. La alteración estructural más frecuente fue la rotura de cuerdas tendíneas, presente en 58 casos (66\%) y el prolapso de velo posterior, que se presentó en 60 casos (68\%). En 40 casos (46\%) se consideró que existía una dilatación significativa del anillo mitral. La Tabla 2 detalla los principales mecanismos de la insuficiencia mitral. En la mayoría de los pacientes existía más de una lesión responsable de ésta.

De acuendo a los tipos de insuficiencia mitral mixomatosa definidos por Carpentier ${ }^{9}$, ésta fue tipo deficiencia fibroelástica en 55 pacientes (62\%), tipo Barlow en 27 (31\%) y marfanoide en 6 (7\%).

Procedimientos reconstructores. El procedimiento más frecuente fue la resección cuadrilateral del velo posterior, en 60 casos (68\%). En 14 casos (16\%) se efectuó una transferencia de cuerdas tendíneas desde el velo posterior al anterior. En la gran mayoría (97\%) estos procedimientos se complementaron con una anuloplastia mitral con anillo de Carpentier-Edwards ${ }^{\mathrm{TM}}$. En 73 casos se utilizó un anillo de Carpentier-Edwards Classic $^{\mathrm{TM}}$ y en 12 el nuevo modelo Physio-Ring (Tabla 3).

En 30 pacientes (34\%) se efectuó un procedimiento asociado. En 21 casos (24\%) éste consistió en una revascularización miocárdica con un promedio de 2 puentes coronarios ( 1 a 4). En 2 casos se efectuó un reemplazo aórtico y en 2 una

\section{Tabla 2. H allazgos anátomo-patológicos intraoperatorios en 88 pacientes con reparación valvular mitral por insuficiencia mitral degenerativa}

\begin{tabular}{|lcc|}
\hline Anatomía patológica & No casos $^{\circ}$ & $\%$ \\
\hline Prolapso velo posterior & 60 & 68 \\
Rotura cuerdas & 58 & 66 \\
Dilatación anillo & 40 & 46 \\
Alargamiento cuerdas & 33 & 38 \\
Prolapso velo anterior & 27 & 31 \\
Otros & 16 & 18 \\
\hline
\end{tabular}


anuloplastia tricuspídea. Finalmente, en 5 casos $(6 \%)$ se efectuaron otros procedimientos.

La ecocardiografía transesofágica intraoperatoria demostró ausencia de insuficiencia mitral en 76 pacientes (86\%) e insuficiencia mitral leve en 12 (14\%).

Mortalidad intrahospitalaria. Dos pacientes $(2,3 \%)$ fallecieron en el período perioperatorio. El primer paciente fue una mujer de 58 años que presentó al tercer día de su operación un accidente vascular encefálico falleciendo al séptimo día. El segundo paciente fue una mujer de 73 años que

\section{Tabla 3. Procedimientos quirúrgicos reconstructores mitrales efectuados en 88 pacientes con insuficiencia mitral degenerativa}

\begin{tabular}{|lcc|}
\hline Procedimientos reconstructores & $\mathrm{N}^{0}$ casos & $\%$ \\
\hline $\begin{array}{l}\text { Resección cuadrilateral } \\
\quad \text { velo posterior }\end{array}$ & 60 & 68 \\
$\begin{array}{l}\text { Transferencia cuerdas } \\
\quad \text { tendíneas }\end{array}$ & 14 & 16 \\
Acortamiento cuerdas & & \\
$\quad$ tendíneas & 6 & 7 \\
Otros & 14 & 16 \\
Anulopatías anillo Carpentier & 85 & 97 \\
\hline
\end{tabular}

falleció súbitamente al undécimo día por un tromboembolismo pulmonar.

Seguimiento. El seguimiento se cerró en agosto de 2002 y se completó en 93\% de los casos, con un promedio de 53,6 meses $\pm 36,2$ meses, un margen de 2,5 a 128,1 meses y una mediana de 41,9 meses. En relación a la sobrevida, el seguimiento fue $100 \%$ puesto que todos los pacientes fueron cotejados por el Registro Civil e Identificación de Chile.

Seis pacientes fallecieron durante el seguimiento. En 3 de éstos la causa de muerte fue cardíaca, la que se presentó en promedio a los 59,5 meses \pm 31 meses (39 a 96 meses). Los otros 3 pacientes fallecieron de causas no cardíacas. La Figura 2 muestra la curva de sobrevida global, incluyendo la mortalidad perioperatoria. Así, la probabilidad de estar vivo a un año fue $98 \% \pm$ $1,6 \%$, a 5 años $88 \% \pm 4,0 \%$ y a 10 años $82 \% \pm$ $6,0 \%$. Por otra parte, la probabilidad de estar libre de muerte cardíaca fue $98 \% \pm 1,6$ a un año, $94 \% \pm 3,0 \%$ a 5 años y $89 \% \pm 6,0 \%$ a 10 años (Figura 3).

Durante este período 2 pacientes $(2,3 \%)$ fueron reoperados. El primero fue un hombre de 74 años quien presentó un desprendimiento del anillo protésico, efectuándose al mes y medio de su operación un reemplazo mitral. El segundo paciente fue una mujer de 59 años que presentó al

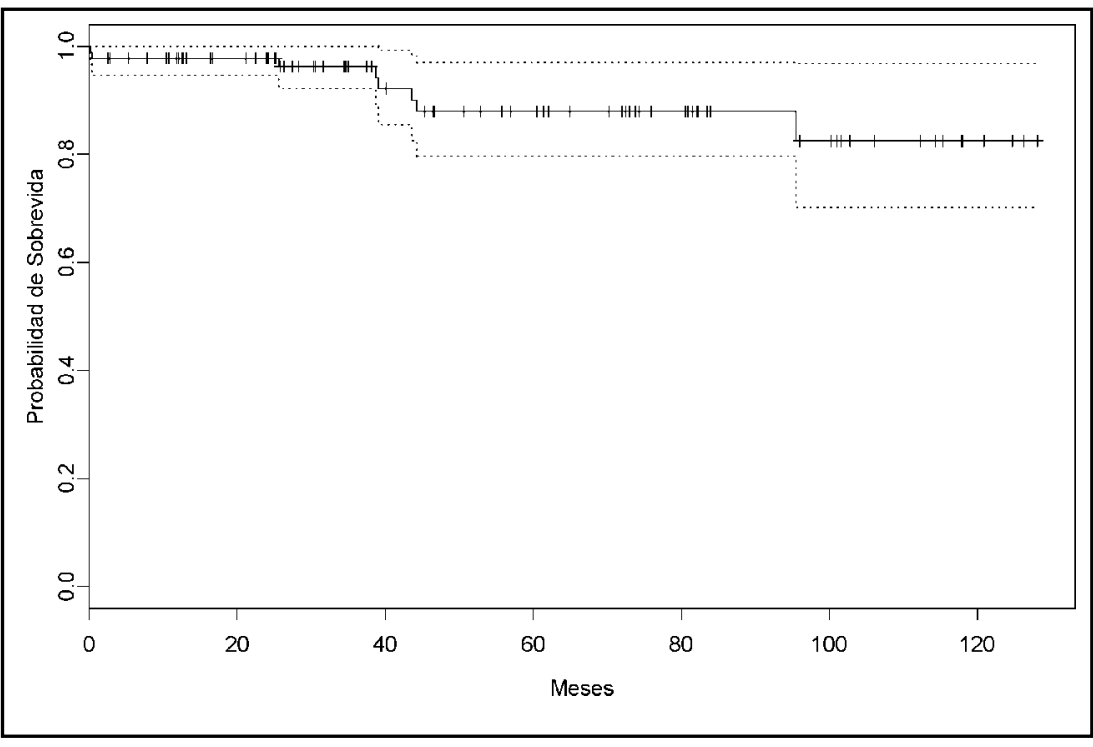

FIgURA 2. Curva de sobrevida actuarial (Kaplan-Meier) global para pacientes con reparación valvular mitral por insuficiencia mitral degenerativa. 
quinto mes una endocarditis del anillo protésico procediéndose a la remoción del anillo. Así, la probabilidad de estar libre de reoperación fue de $98 \% \pm 1,6 \%$ a 1,5 y 10 años (Figura 4 ).

Por otra parte, durante el seguimiento 3 pacientes fueron rehospitalizados por causa cardíaca. Otros 24 pacientes requirieron rehospitalización por causas no cardíacas. La Figura 5 muestra la probabilidad de estar libre de eventos, incluyendo muerte cardíaca, reoperación y re- hospitalización cardiovascular, la que fue $94 \% \pm$ $2 \%$ a 1 año, $90 \% \pm 3 \%$ a 5 años y $84 \% \pm 6 \%$ a 10 años.

Todos los pacientes vivos al momento del cierre del seguimiento presentaban mejoría de su $\mathrm{CF}$, la que disminuyó en promedio de $2,6 \pm 0,9$ a $1,2 \pm 0,4$ ( $p<0,001)$, estando $68 \%$ de éstos en CF I y $32 \%$ en II. La Figura 6 compara la CF previo a la reparación con la CF alejada post reparación mitral.


FiguRa 3. Curva de probabilidad actuarial (KaplanMeier) de estar libre de muerte de causa cardíaca para pacientes con reparación valvular mitral por insuficiencia mitral degenerativa.
FIguRA 4. Curva de probabilidad actuarial (KaplanMeier) de estar libre de reoperación para pacientes con reparación valvular mitral por insuficiencia mitral degenerativa. 
El $23 \%$ de los pacientes se encontraba sin tratamiento medicamentoso al término del seguimiento. La Tabla 4 detalla los medicamentos utilizados en los otros pacientes. Como se observa en ésta, 21 pacientes (26\%) se encontraban en tratamiento anticoagulante, por fibrilación auricular.

Ningún paciente presentó complicaciones hemorrágicas durante el seguimiento. Dos pacientes presentaron un accidente isquémico cerebral transitorio. Como se señaló, sólo una paciente presentó una endocarditis mitral, por lo que la
Tabla 4. Medicamentos utilizados por $77 \%$ de los pacientes que se encontraban al término del período de seguimiento con tratamiento médicamentoso.

\begin{tabular}{|lcc|}
\hline Medicamentos & No casos & $\%$ \\
\hline Diuréticos & 28 & 35 \\
Vasodilatadores & 26 & 33 \\
Anticoagulantes & 21 & 26 \\
Antiarrítmicos & 18 & 21 \\
Digitálicos & 17 & 21 \\
\hline
\end{tabular}

FIgURA 5. Curva de probabilidad actuarial (KaplanMeier) de estar libre de eventos cardiovasculares para pacientes con reparación mitral por insuficiencia mitral degenerativa.

FIguRA 6. Capacidad funcional pre y post reparación valvular mitral alejada por insuficiencia mitral degenerativa.
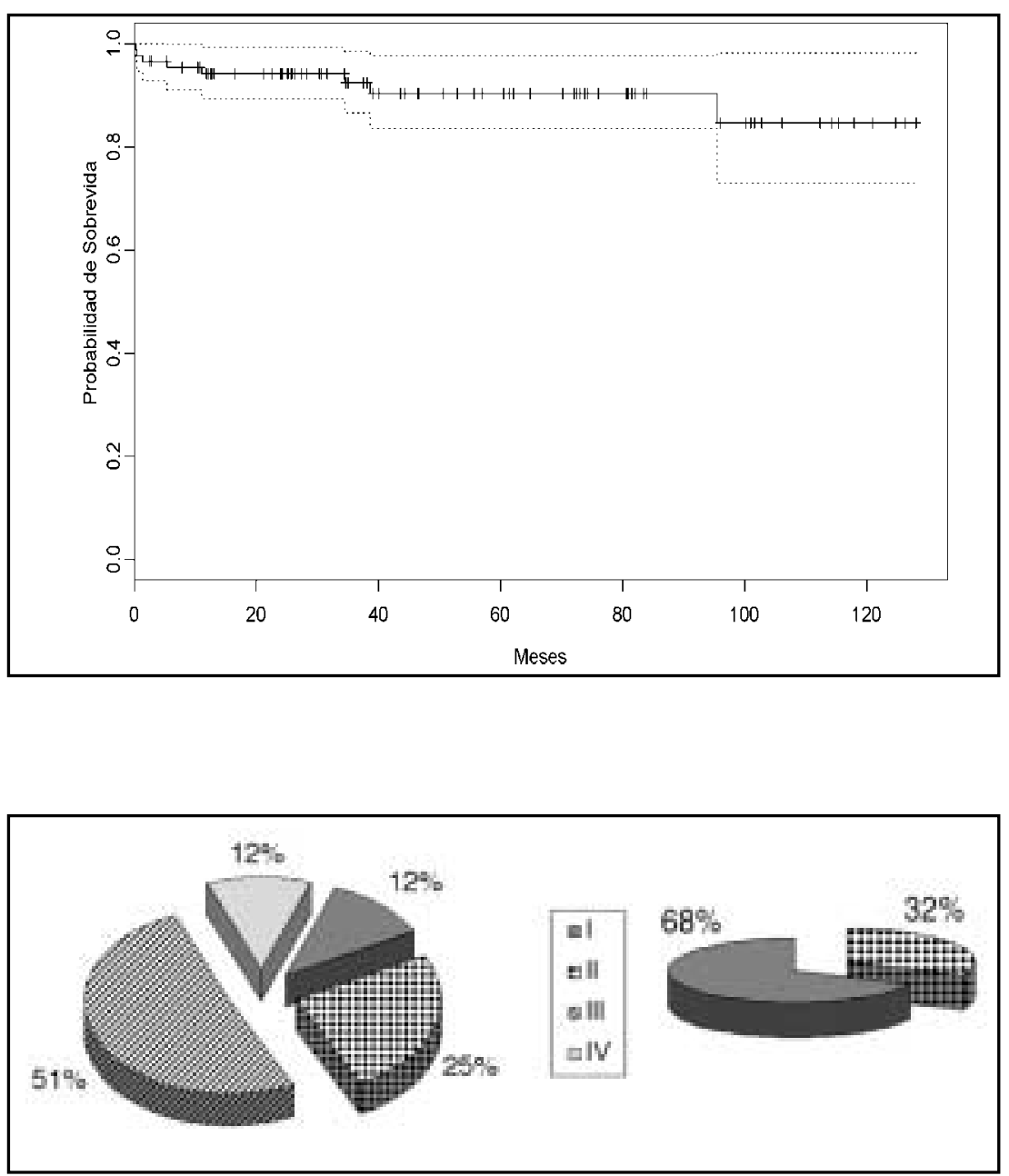
Tabla 5. Parámetros ecocardiográficos pre y post reparación mitral alejados por insuficiencia mitral degenerativa

\begin{tabular}{|lccr|}
\hline Ecocardiografía & Pre reparación & Post reparación & \multicolumn{1}{c|}{$\mathrm{p}$} \\
\hline Diámetro auricular izquierdo & $55 \pm 11 \mathrm{~mm}$ & $47 \pm 8,1 \mathrm{~mm}$ & 0,007 \\
Diámetro diastólico ventricular izquierdo & $61 \pm 7,6 \mathrm{~mm}$ & $54 \pm 8 \mathrm{~mm}$ & $<0,001$ \\
Diámetro sistólico ventricular izquierdo & $37 \pm 9,5 \mathrm{~mm}$ & $37 \pm 9 \mathrm{~mm}$ & $\mathrm{NS}$ \\
Fracción de acortamiento & $41 \pm 9 \%$ & $31 \pm 11 \%$ & $<0,001$ \\
Presión sistólica arteria pulmonar & $47 \pm 10 \mathrm{~mm} \mathrm{Hg}$ & $32 \pm 12 \mathrm{~mm} \mathrm{Hg}$ & 0,002 \\
\hline
\end{tabular}

probabilidad de estar libre de endocarditis a 10 años fue $99 \% \pm 1,2 \%$.

El control ecocardiográfico alejado, disponible en $96,5 \%$ de los casos, con un promedio de $16,5 \pm$ 33,2 meses, demostró ausencia de insuficiencia mitral o solo indicios en $83 \%$ de los casos y una insuficiencia leve en $17 \%$. El diámetro auricular izquierdo promedio fue $47 \mathrm{~mm} \pm 8,1 \mathrm{~mm}$. El diámetro diastólico ventricular izquierdo fue $54 \mathrm{~mm}$ $\pm 8 \mathrm{~mm}$ y el sistólico $37 \mathrm{~mm} \pm 9 \mathrm{~mm}$. La fracción de acortamiento promedio fue $31 \pm 11 \%$. La presión sistólica de arteria pulmonar fue en promedio 32 $\mathrm{mm} \mathrm{Hg} \pm 12 \mathrm{~mm} \mathrm{Hg}$. La Tabla 5 compara parámetros ecocardiográficos pre y post reparación mitral alejados. Con excepción del diámetro sistólico ventricular izquierdo, que no experimentó variación, y la fracción de acortamiento que disminuyó en forma significativa, todos los otros parámetros mejoraron significativamente.

\section{DisCUSIÓN}

A partir de los trabajos pioneros de Carpentier, la cirugía reparadora de la válvula mitral se ha impuesto como el procedimiento de elección para la insuficiencia mitral, llegando incluso a cambiar las indicaciones habituales de ésta ${ }^{7,9-11}$. En gran medida esto también ha sido consecuencia del desarrollo de la ecocardiografía transesofágica, que ha permitido efectuar un acucioso análisis anatómico y funcional pre y post reparación de la válvula mitral ${ }^{1,12,13}$. Por otra parte, en los últimos años se ha observado que el grupo de pacientes en los cuales es más factible efectuar una reparación mitral exitosa son aquellos portadores de una insuficiencia mitral degenerativa, los que serían también los que más se beneficiarían con ésta1,14,15. Por último, es este grupo de pacientes en los cuales es posible predecir mejor con la ecocardiografía la probabilidad de reparar una válvula mitral insuficiente. En la experiencia del autor $^{3}$, la presencia de cuerdas tendíneas rotas, independientemente si son del velo anterior 0 posterior, predice certeramente la probabilidad de éxito de la intervención. Una muestra de lo anterior es que del total de 166 pacientes con reparación valvular mitral, más de la mitad tenían una insuficiencia mitral degenerativa y de éstos, dos tercios cuerdas rotas.

Al igual como ha sucedido en otros centros cardiovasculares, la reparación mitral se impuso en el nuestro progresivamente por sobre el reemplazo valvular ${ }^{1,16}$. Más aún, los beneficios observados a largo plazo de la reparación mitral junto a la baja mortalidad operatoria, han modificado sustancialmente la indicación quirúrgica de la insuficiencia mitral, al punto que actualmente la presencia de una insuficiencia significativa, junto a la probabilidad de poder reparar la válvula mitral es de por sí indicación quirúrgica ${ }^{1,12,17}$. En nuestra experiencia esto se ha traducido en que más de un tercio de nuestros pacientes estaban asintomáticos o poco sintomáticos.

Hallazgos intraoperatorios y procedimientos reconstructores. Al igual que la mayoría de las series, la lesión estructural más frecuente fue la rotura de cuerdas tendíneas, en especial del velo posterior la que, como se ha señalado, es un predictor ecocardiográfico del éxito de la reparación, ya que permite precisar el principal meca- 
nismo de la insuficiencia mitral. Por lo mismo, el procedimiento más frecuente consistió en la resección cuadrilateral del velo posterior (68\%), efectuado con técnica de "plicatura" del anillo posterior o con técnica de "deslizamiento", en casos de valvulopatía mitral tipo Barlow 7,9,18,19. Como ha sido nuestra preferencia, casi todos estos procedimientos se complementaron con una anuloplastia con anillo de Carpentier-Edwards $^{\mathrm{TM}}$, la que pretende remodelar el anillo mitral devolviéndole su forma original, que se asemeja a un riñón, y con esto disminuir el diámetro anteroposterior, aumentando la coaptación de los velos mitrales a la vez que reforzar las suturas anulares y prevenir futuras dilataciones. El procedimiento asociado más frecuente, presente en casi la cuarta parte de los pacientes, fue la revascularización miocárdica. La enfermedad coronaria fue concomitante con la valvulopatía mitral degenerativa y la insuficiencia mitral secundaria sólo a ésta última ${ }^{21}$.

Ya a fines de la década 1980-89, se observó que uno de los factores predisponentes a fracaso de la reparación mitral era la insuficiencia residual de cierta significación (2 o más +$)^{14,22}$. De aquí que $86 \%$ de nuestros pacientes abandonó el pabellón de cirugía sin insuficiencia mitral y los restantes, con una insuficiencia leve. Para esto, el cirujano se esforzó en la evaluación intraoperatoria de la válvula mitral y a su vez el ecocardiografista fue muy acucioso en su examen.

Mortalidad intrahospitalaria. Como en la mayoría de las series publicadas, la mortalidad operatoria fue baja, de 2,3\%1,14,15,23. Esta no debe ser explicada por un supuesto menor riesgo 0 porque la reparación sea un procedimiento más simple que el reemplazo mitral. De hecho es todo lo contrario, puesto que son los pacientes más graves los que más se benefician de una reparación mitral con mantención de la integridad del aparato valvular y por consiguiente, de una mejor preservación de la estructura y función del ventrículo izquierdo. Igualmente, la reconstrucción de la válvula mitral, si bien en la actualidad es un procedimiento completamente estandarizado, susceptible de ser enseñado y aprendido, es técnicamente más demandante, requiriendo casi siempre períodos de circulación extracorpórea e isquemia miocárdica prolonga$\operatorname{dos}^{1,14,15,23}$.

Seguimiento. En esta experiencia, con un promedio de seguimiento de 54 meses y un máximo de 128, sólo 3 pacientes fallecieron de causa cardíaca, por lo que la probabilidad de estar libre de muerte cardiovascular fue excelente, de $94 \%$ a 5 años y $89 \%$ a 10 años. Igualmente, sólo 2 pacientes requirieron ser reoperados, uno de ellos por una falla quirúrgica precoz y la otra por una endocarditis del anillo protésico, en la cual no fue necesario efectuar un reemplazo mitral. De aquí que la probabilidad de estar libre de reoperación haya sido para todo el seguimiento de $98 \% \pm 1,6 \%$. Asimismo, la probabilidad de estar libre de eventos cardiovasculares, incluyendo muerte perioperatoria y cardíaca, reoperación y rehospitalización por causa cardiovascular, fue muy satisfactoria, $90 \%$ a 5 años y $84 \%$ a 10 años.

Del mismo modo, no sólo la sobrevida fue muy satisfactoria sino que también hubo un impacto significativo en la CF de los pacientes, la que disminuyó de $2,6 \pm 0,9$ a $1,2 \pm 0,4$, estando $68 \%$ en CF I y $32 \%$ en II. Además, la gran mayoría de los pacientes se encontraba sin tratamiento anticoagulante y los que lo estaban era por fibrilación auricular, lo que es una situación distinta a requerir anticoagulación por una prótesis intracardíaca. La probabilidad de estar libre de endocarditis a 10 años fue excelente, de $99 \% \pm 1,2 \%$. Por último, hubo una clara y sostenida mejoría en los parámetros ecocardiográficos alejados, tanto en cuanto a insuficiencia mitral como a los diámetros auriculares y ventriculares diastólicos izquierdos así como una disminución de la presión de arteria pulmonar. Como era predecible, el diámetro sistólico ventricular izquierdo se mantuvo igual y la fracción de acortamiento se redujo significativamente, lo que se explica por el aumento de la post carga que significa la corrección de la insuficiencia mitral. Estos resultados son muy similares a los publicados en la literatura internacional $1,10,11,14,15,18$

Limitaciones del estudio. Este es un estudio observacional descriptivo de pacientes operados sucesi- 
vamente en un período de 10 años y como tal tiene las limitaciones de los trabajos no aleatorios ni doble ciego. Sin embargo, los resultados son muy satisfactorios y pueden ser comparados con series históricas de reemplazo mitral para dar mayor validez a los mismos ${ }^{1,16,24,26}$.

\section{REFERENCIAS}

1. Отто CM. Evaluation and management of chronic mitral regurgitation. N Engl J Med 2001; 345: 7406.

2. Zalaquett R. Cirugía reparadora de la insuficiencia valvular mitral. Rev Chil Cir 1994; 46: 127-36.

3. Zalaquett R, Morán S, Irarrázaval MJ, Maturana G, Braun S, Chamorro G et aL. Reconstrucción válvula mitral. Cirugía reparadora de la insuficiencia valvular mitral con evaluación ecocardiográfica transesofágica intraoperatoria. Rev Chil Cardiol 1996; 15: 78-84.

4. Zalaquett R, Chamorro S, Braun S, Garrido L, Howard M, Morán S et al. Resultados alejados de la cirugía reconstructora de la insuficiencia mitral. Rev Méd Chile 1999; 127: 1093-100.

5. Zalaquett $R$, Becker P, Irarrázaval MJ, Morán S, Maturana G, Navarro M et al. Cardioplegia retrógrada por canulación transauricular derecha del seno coronario. Rev Chil Cardiol 1993; 12: 68-71.

6. Zalaquett R, Morán S, Irarrázaval MJ, Maturana G, NAVARRO M, Braun S et al. Acceso auricular izquierdo transeptal y superior combinado. Rev Chil Cir 1993; 45: 225-8.

7. Carpentier A. Cardiac valve surgery, The "French correction". J Thorac Cardiovasc Surg 1983; 86: 323-37.

8. Edmunds LH, Ciark RE, Cohn LH, Miler DC, Weisel $\mathrm{RD}$. Guidelines for reporting morbidity and mortality after cardiac valvular operations. J Thorac Cardiovasc Surg 1988; 96: 351-3.

9. Carpentier A, Reliand J, Deloche A, Fabián J, D’Allaines C, Blondeau P et al. Conservative management of the prolapsed mitral valve. Ann Thorac Surg 1978; 26: 394-402.

10. Cosgrove DM, Chávez AM, Lytle BW, Gill CC, Steward RW, Taylor PC et al. Result of mitral valve reconstruction. Circulation 1986; 74 (suppl): I 82-7.
Conclusiones. Estos buenos resultados, tanto clínicos como ecocardiográficos, permiten sostener que la cirugía reparadora es una excelente alternativa para la corrección de la insuficiencia mitral degenerativa.

11. Galloway AC, Colvin SB, Baumann G, Espósito R, VOHRA R, HARTY S ET AL. Long term results of mitral valve reconstruction with Carpentier technique in 148 patients with insuficiency. Circulation 1988; 78 (suppl): 197-205.

12. Enríquez-Sarano M, Freeman WK, Tribouiloy CM, Orszulak TA, Khandheria BK, Seward JB et aL. Functional anatomy of mitral regurgitation: Accuracy and outcome implications of transesophageal echocardiography. J Am Coll Cardiol 1999; 34: 1129-36.

13. Chamorro G, Braun S, Fernández MS, Zalaquett R, LóPEz G. Monitorización de la reparación valvular mitral con ecografía transesofágica intraoperatoria. Rev Chil Cardiol 1992; 11: 201.

14. Cosgrove D. Surgery for degenerative mitral valve disease. Semin Thorac Cardiovasc Surg 1989; 1: 183-93.

15. David TE, Armstrong S, Sub Z, Daniel L Late results of mitral valve repair for mitral regurgitation due to degenerative disease. Ann Thorac Surg 1994; 56: 7-14.

16. Braunwald E. Valvular heart disease. En: Braunwald E, ed. Heart Disease. Philadelphia; Editorial WB Saunders Company 1988; 1045.

17. Tribouiloy CM, Enríquez-Sarano M, SchafF HV, Orszulak TA, Bailey KR, Tajik J et al. Impact of preoperative symptoms on survival after surgical correction of organic mitral regurgitation. Rationale for optimizing surgical indications. Circulation 1999; 99: 400-5.

18. Jebara VA, Mihaileanvu S, Acar C, Brizard C, Grace P, Latremouile $C$ et al. Left ventricular outflow tract obstruction after mitral valve repair. Results of the sliding leaflet technique. Circulation 1993; 88: (supp II) II30-4.

19. BarLow JB, Рососк WA. Billowing, floppy, prolapsed or flail mitral valves? Am J Cardiol 1985; 55: 501-2.

20. Carpentier AF, Lessana A, Relland JY, Bew E, 
Mihaileanu S, BerReBi AJ et al. The "Physio-Ring": An advanced concept in mitral valve annuloplasty. Ann Thorac Surg 1995; 60: 1177-85.

21. Zalaquett R, Garrido L, Morán S, Irarrázaval MJ, Becker P, Maturana G et al. Cirugía de la insuficiencia mitral isquémica. Rev Méd Chile 2002; 130: 9-16.

22. Smedira NG, Selman R, Cosgrove DM, McCarthy PM, LtLe BW, TAYLOR PC et al. Repair of anterior leaflet prolapse: Chordal transfer is superior to chordal shortening. J Thorac Cardiovasc Surg 1996; 112: 287-91.

23. Cosgrove DM, Stewart WJ. Mitral valvuloplasty. Curr Probl Cardiol 1989; 14: 353-416.

24. Galloway AC, Colvin SB, Baumann FG, Grossi EA, Ribakove GH, HaRTy S et al. A comparison of mitral valve reconstruction with mitral valve replacement: intermediate term results. Ann Thorac Surg 1989; 47: 655-62.

25. Grossi EA, Galoway aC, Miuer JS, Ribakove GH, CuLford AT, Espóstio R ET AL. Valve repair versus replacement for mitral insufficiency: when is a mechanical valve still indicated? J Thorac Cardiovasc Surg 1998; 115: 389-96.

\section{Agradecimientos}

Los autores expresan su sincero agradecimiento a las bioestadísticas señoritas Paola Viviani y Alessandra Gederlini y a su secretaria señorita Verónica Delgado, por su asistencia estadística y las múltiples correcciones del manuscrito. 